\title{
Approximate Solutions of Stochastic Differential Delay Equations with Markovian Switching *
}

\author{
Xiaoyue $\mathrm{Li}^{1,2} \quad$ Xuerong $\mathrm{Mao}^{3 \dagger} \quad \mathrm{Yi} \mathrm{Shen}^{4}$
}

1. Mathematics school, Jilin University, Changchun, 130012, P. R. China.

2. School of Mathematics and Statistics, Northeast Normal University, Changchun, 130024, China.

3. Department of Statistics and Modelling Science, University of Strathclyde, Glasgow, G1 1XH, UK.

4. Department of Control Science and Engineering, Huazhong University of Science and Technology, Wuhan, Hubei, 430074, China.

\begin{abstract}
Our main aim is to develop the existence theory for the solutions to stochastic differential delay equations with Markovian switching (SDDEwMSs) and to establish the convergence theory for the Euler-Maruyama approximate solutions under the local Lipschitz condition. As an application, our results are used to discuss a stochastic delay population system with Markovian switching.
\end{abstract}

Keywords. Euler-Maruyama method; Generalized Itô's formula; Brownian motion; Markov chain.

2000 MR Subject Classification. 60H10, 34K50, 34K20.

${ }^{*}$ Research supported by the National Natural Science Foundation of China (10571021; 10671031; 10701020; 60574025; 60740430664), Key Project of Chinese Ministry of Education (106062) and Key Laboratory for Applied Statistics of MOE (KLAS).

$\dagger$ Corresponding author. E-mail address: xuerong@stams.strath.ac.uk 


\section{Introduction}

The hybrid systems driven by continuous-time Markov chains have been used to model many practical systems where they may experience abrupt changes in their structure and parameters caused by phenomena such as component failures or repairs changing subsystem interconnections, and abrupt environmental disturbances. For example, in his book [8], Mariton explained that the hybrid systems had been emerging as a convenient mathematical framework for the formulation of various design problems in different fields such as evasive target tracking, fault tolerance detection, and manufacturing processes. Recently, this hybrid approach had been extended to population dynamics (see e.g. [4, 12]) and the hybrid population systems are illustrated as a switching between two or more regimes of environment, which differ by factors such as nutrition or as rain falls $[1,11]$.

One of the important classes of the hybrid systems is the stochastic differential delay equation with Markovian switching (SDDEwMS)

$$
d x(t)=f(x(t), x(t-\tau), r(t)) d t+g(x(t), x(t-\tau), r(t)) d B(t) .
$$

Here the state vector has three components $x(t), x(t-\tau)$ and $r(t)$ : the first two ones are in general referred to as the current and past states while the third one is regarded as the mode. In its operation, the system switches from one mode to another in a random way, and the switching between the modes is governed by a Markov chain. For details of SDDEwMSs, the reader is referred to $[7,10]$ among others.

Our primary objective is to study the existence of the exact solutions to the SDDEwMS (1.1) and the convergence problem for the Euler-Maruyama (EM) approximations under some relax conditions. The existence theory of the exact solutions to the SDDEwMS (1.1) has been studied quite well. In order to have a unique global (i.e. no explosion in a finite time) solution for any given initial data, the coefficients of the equation are usually required to satisfy the local Lipschitz condition and the linear growth condition (see e.g. [7]). Unfortunately, the linear growth condition is often not met by many systems in practice. For example, consider the stochastic hybrid delay population system

$d x(t)=\operatorname{diag}\left(x_{1}(t), \ldots, x_{n}(t)\right)[(b(r(t))+A(r(t)) x(t)+B(r(t)) x(t-\tau)) d t+\sigma(r(t)) d B(t)]$, 
where

$$
x=\left(x_{1}, \cdots, x_{n}\right)^{T} \in \mathbb{R}^{n}, \quad b \in \mathbb{R}^{n}, \quad A \in \mathbb{R}^{n \times n}, \quad B \in \mathbb{R}^{n \times n}, \quad \sigma \in \mathbb{R}^{n \times m}
$$

and $B(t)$ is an $m$-dimensional Brownian motion. It is straightforward to see that the linear growth condition is not satisfied by this system. It is in this spirit that Mao et al. [6] have recently established a more general existence theory for the solution. In this paper we will establish an alternative theorem on the existence and uniqueness of the solution under the local Lipschitz condition plus a Lyapunov-type condition.

Moreover, most of the SDDEwMSs do not have explicit solutions whence the numerical solutions are required. The classical convergence theory for numerical methods to SDEs requires the coefficients of the equations to be globally Lipschitz, see [3], [5], for example. Recently, Highham et al. [2] released the global Lipschitz condition but required the linear growth condition or the bounded $p$ th moment property of both exact and approximate solutions. These conditions are somehow still too restrictive, for example, they are not satisfied by the population system (1.2). On the other hand, Marion et al. [9] proved that the numerical solutions based on the Euler-Maruyama (EM) scheme will converge to the true solutions for a broad class of SDEs without the linear growth condition nor the bounded $p$ th moment property under some additional conditions in terms of Lyapunov-type functions. All these results on numerical solutions are about SDEs and it is non-trivial to develop them for the SDDEwMSs as they are more complicated.

In this paper we borrow the ideas from [6] and [9] and apply them to the SDDEwMS (1.1). We will establish a new theorem on the existence and uniqueness of the solution under the local Lipschitz condition plus a Lyapunov-type condition in section 3. Under these conditions as well as the local Lipschitz continuity of the derivatives of the Lyapunov function, we will then show the convergence in probability of the EM solutions to the exact solution in section 4. Finally, we will apply our new results to study the population system (1.2) in section 5.

\section{Notations}

Throughout this paper, we will use the following notations. We let $\left(\Omega, \mathcal{F},\left\{\mathcal{F}_{t}\right\}_{t \geq 0}, \mathbb{P}\right)$ be a complete probability space with a filtration $\left\{\mathcal{F}_{t}\right\}_{t \geq 0}$ satisfying the usual conditions (i.e. it is increasing and right continuous while $\mathcal{F}_{0}$ contains all $\mathbb{P}$-null sets). Let $B(t)=$ 
$\left(B_{1}(t), \cdots, B_{m}(t)\right)^{T}$ be an $m$-dimensional Brownian motion defined on this probability space. Let $C(X, Y)$ denote the family of all continuous mappings from the topological space $X$ to the topological space $Y$. Let $\tau>0$ and $C_{\mathcal{F}_{t}}\left([-\tau, 0] ; \mathbb{R}^{n}\right)$ be the family of all $\mathcal{F}_{t}$-measurable, $C\left([-\tau, 0] ; \mathbb{R}^{n}\right)$-valued random variables $\xi=\{\xi(\theta):-\tau \leq \theta \leq 0\}$. Let $C_{\mathcal{F}_{t}}^{b}\left([-\tau, 0] ; \mathbb{R}^{n}\right)$ denote the family of all bounded random variables $\xi \in C_{\mathcal{F}_{t}}\left([-\tau, 0] ; \mathbb{R}^{n}\right)$. Also let $L_{\mathcal{F}_{t}}^{2}\left([-\tau, 0] ; \mathbb{R}^{n}\right)$ denote the family of all random variables $\xi \in C_{\mathcal{F}_{t}}\left([-\tau, 0] ; \mathbb{R}^{n}\right)$ such that

$$
\|\xi\|_{\mathbb{E}}^{2}:=\sup _{-\tau \leq \theta \leq 0} \mathbb{E}|\xi(\theta)|^{2}<\infty
$$

Let $r(t)$ be a right-continuous Markov chain on the probability space taking values in a finite state space $\mathbb{S}=\{1,2, \ldots, N\}$ with the generator $\Gamma=\left(\gamma_{u v}\right)_{N \times N}$ given by

$$
\mathbb{P}\{r(t+\delta)=v \mid r(t)=u\}= \begin{cases}\gamma_{u v} \delta+o(\delta), & \text { if } u \neq v \\ 1+\gamma_{u v} \delta+o(\delta), & \text { if } u=v\end{cases}
$$

where $\delta>0$. Here $\gamma_{u v}$ is the transition rate from $u$ to $v$ and $\gamma_{u v} \geq 0$ if $u \neq v$ while

$$
\gamma_{u u}=-\sum_{v \neq u} \gamma_{u v}
$$

We assume that the Markov chain $r(\cdot)$ is independent of the Brownian motion $B(\cdot)$. Let $L_{\mathcal{F}_{t}}(\Omega ; \mathbb{S})$ denote the family of all $\mathcal{F}_{t}$-measurable $\mathbb{S}$-valued random variables.

Consider the stochastic differential delay equation with Markovian switching (SDDEwMS) of the form

$$
d x(t)=f(x(t), x(t-\tau), r(t)) d t+g(x(t), x(t-\tau), r(t)) d B(t)
$$

on $t \geq 0$ with initial data $\{x(t):-\tau \leq t \leq 0\}=\xi \in C_{\mathcal{F}_{0}}\left([-\tau, 0] ; \mathbb{R}^{n}\right), r(0)=r_{0} \in$ $L_{\mathcal{F}_{0}}(\Omega ; \mathbb{S})$ and

$$
f: \mathbb{R}^{n} \times \mathbb{R}^{n} \times \mathbb{S} \rightarrow \mathbb{R}^{n}, \quad g: \mathbb{R}^{n} \times \mathbb{R}^{n} \times \mathbb{S} \rightarrow \mathbb{R}^{n \times m}
$$

Moreover, let $C^{2}\left(\mathbb{R}^{n} \times \mathbb{S} ; \mathbb{R}_{+}\right)$denote the family of all nonnegative functions $V(x, i)$ on $\mathbb{R}^{n} \times \mathbb{S}$ which are continuously twice differentiable in $x$. For each $V \in C^{2}\left(\mathbb{R}^{n} \times \mathbb{S} ; \mathbb{R}_{+}\right)$, define an operator $\mathcal{L} V$ from $\mathbb{R}^{n} \times \mathbb{R}^{n} \times \mathbb{S}$ to $\mathbb{R}$ by

$$
\begin{aligned}
\mathcal{L} V(x, y, i)= & V_{x}(x, i) f(x, y, i) \\
& +\frac{1}{2} \operatorname{trace}\left[g^{T}(x, y, i) V_{x x}(x, i) g(x, y, i)\right] \\
& +\sum_{j=1}^{N} \gamma_{i j} V(x, j) .
\end{aligned}
$$


where

$$
V_{x}(x, i)=\left(\frac{\partial V(x, i)}{\partial x_{1}}, \ldots, \frac{\partial V(x, i)}{\partial x_{n}}\right), \quad V_{x x}(x, i)=\left(\frac{\partial^{2} V(x, i)}{\partial x_{i} \partial x_{j}}\right)_{n \times n}
$$

Given a step size $\Delta>0$, let $X(t)$ be the continuous EM approximate solution to the SDDEwMS (2.1). This was defined in [7] but we recall here its detailed definition. First of all, let us explain how to simulate the discrete-time Markov chain $\left\{r_{k}^{\Delta}\right\}$. Recall the property of the embedded discrete-time Markov chain: Given a step size $\Delta>0$, let $r_{k}^{\Delta}=r(k \Delta)$ for $\Delta>0$ and $k \geq 0$. Then $\left\{r_{k}^{\Delta}, k=0,1,2, \cdots\right\}$ is a discrete-time Markov chain with the one-step transition probability matrix

$$
\mathbb{P}(\Delta)=\left(\mathbb{P}_{i j}(\Delta)\right)_{N \times N}=e^{\Delta \Gamma}
$$

The discrete-time Markov chain $\left\{r_{k}^{\Delta}, k=0,1,2, \cdots\right\}$ can be simulated as follows: compute the one-step transition probability matrix

$$
\mathbb{P}(\Delta)=\left(\mathbb{P}_{i j}(\Delta)\right)_{N \times N}=e^{\Delta \Gamma}
$$

Let $r_{0}^{\Delta}=r_{0}$ and generate a random number $\zeta_{1}$ which is uniformly distributed in [0,1]. If $\zeta_{1}=1$ then let $r_{1}^{\Delta}=r_{1}=N$ or otherwise find the unique integer $r_{1} \in \mathbb{S}$ for

$$
\sum_{j=1}^{r_{1}-1} \mathbb{P}_{r_{0}, j}(\Delta) \leq \zeta_{1}<\sum_{j=1}^{r_{1}} \mathbb{P}_{r_{0}, j}(\Delta)
$$

and let $r_{1}^{\Delta}=r_{1}$, where we set $\sum_{j=1}^{0} \mathbb{P}_{r_{0}, j}(\Delta)=0$ as usual. Generate independently a new random number $\zeta_{2}$ which is again uniformly distributed in $[0,1]$. If $\zeta_{2}=1$ then let $r_{2}^{\Delta}=r_{2}=N$ or otherwise find the unique integer $r_{2} \in \mathbb{S}$ for

$$
\sum_{j=1}^{r_{2}-1} \mathbb{P}_{r_{1}, j}(\Delta) \leq \zeta_{2}<\sum_{j=1}^{r_{2}} \mathbb{P}_{r_{1}, j}(\Delta)
$$

and let $r_{2}^{\Delta}=r_{2}$. Repeating this procedure a trajectory $\left\{r_{k}^{\Delta}, k=0,1,2, \cdots\right\}$ can be generated. This procedure can be carried out independently to obtain more trajectories.

After explaining how to simulate the discrete-time Markov chain $\left\{r_{k}^{\Delta}\right\}$, we can now define the EM approximate solution to equation (2.1). To cope with the time lag, we choose a step size $\Delta>0$ to be a fraction of $\tau$, whence $\bar{k}:=\tau / \Delta$ is a positive integer. Let $t_{k}=k \Delta$ for $k \geq-\bar{k}$. Set $X_{k}=\xi\left(t_{k}\right)$ for $k=-\bar{k},-\bar{k}+1, \cdots, 0$ and then compute the discrete approximations $X_{k} \approx x\left(t_{k}\right)$ for $k \geq 1$ by

$$
X_{k+1}=X_{k}+f\left(X_{k}, X_{k-\bar{k}}, r_{k}^{\Delta}\right) \Delta+g\left(X_{k}, X_{k-\bar{k}}, r_{k}^{\Delta}\right) \Delta B_{k}
$$


where $\Delta B_{k}=B\left(t_{k+1}\right)-B\left(t_{k}\right)$. Let

$$
\bar{r}(t)=r_{k}^{\Delta} \quad \text { for } t \in\left[t_{k}, t_{k+1}\right), \quad k \geq 0
$$

and

$$
\bar{X}(t)=X_{k} \quad \text { for } t \in\left[t_{k}, t_{k+1}\right), \quad k \geq-\bar{k} .
$$

Define the continuous EM approximate solution by setting $X(t)=\xi(t)$ for $t \in[-\tau, 0]$ and forming

$$
X(t)=X_{0}+\int_{0}^{t} f(\bar{X}(s), \bar{X}(s-\tau), \bar{r}(s)) d s+\int_{0}^{t} g(\bar{X}(s), \bar{X}(s-\tau), \bar{r}(s)) d B(s)
$$

for $t \geq 0$. Note that $X\left(t_{k}\right)=\bar{X}\left(t_{k}\right)=X_{k}$ for $k \geq-\bar{k}$, that is $X(t)$ and $\bar{X}(t)$ coincide with the discrete solution at the grid-points.

Before we close this section, let us impose the following hypotheses:

Assumption 1 Assume that both $f$ and $g$ satisfy the local Lipschitz condition. That is for each $R>0$ there is an $L_{R}>0$ such that

$$
\left|f\left(x_{1}, y_{1}, i\right)-f\left(x_{2}, y_{2}, i\right)\right| \vee\left|g\left(x_{1}, y_{1}, i\right)-g\left(x_{2}, y_{2}, i\right)\right| \leq L_{R}\left(\left|x_{1}-x_{2}\right|+\left|y_{1}-y_{2}\right|\right)
$$

for all $i \in S$ and those $x_{1}, x_{2}, y_{1}, y_{2} \in \mathbb{R}^{n}$ with $\left|x_{1}\right| \vee\left|x_{2}\right| \vee\left|y_{1}\right| \vee\left|y_{2}\right| \leq R$

Assumption 2 Assume also there is a positive constant $K$ such that the initial data $\xi$ obeys

$$
\mathbb{E}|\xi(u)-\xi(v)|^{2} \leq K|u-v|, \quad-\tau \leq u<v \leq 0 .
$$

\section{Exact Solution}

Let us begin with a result on the existence of the unique global solutions under the local Lipschitz condition and some additional conditions in terms of Lyapunov-type functions.

Theorem 3.1 Let Assumption 1 hold. Assume that there exists a function $V \in C^{2}\left(\mathbb{R}^{n} \times\right.$ $\left.S ; \mathbb{R}_{+}\right)$, a constant $h>0$ and $H \in C\left(\mathbb{R}^{n} ; \mathbb{R}_{+}\right)$such that

$$
\lim _{|x| \rightarrow \infty} V(x, i)=\infty, \quad \forall i \in \mathbb{S}
$$




$$
\mathcal{L} V(x, y, i) \leq h\left(1+V(x, i)+\min _{j \in S} V(y, j)\right)-H(x)+H(y), \quad \forall(x, y, i) \in \mathbb{R}^{n} \times \mathbb{R}^{n} \times \mathbb{S}
$$

Assume furthermore that the initial data $\xi$ obeys that

$$
\mathbb{E} V\left(\xi(0), r_{0}\right)<\infty, \quad \sup _{-\tau \leq t \leq 0} \mathbb{E}\left[\min _{j \in S} V(\xi(t), j)\right]<\infty, \quad \sup _{-\tau \leq t \leq 0} \mathbb{E} H(\xi(t))<\infty .
$$

Then there exists a unique global solution $x(t)$ to the SDDEwMS (2.1) on $[-\tau, \infty)$.

Proof. Assumption 1 guarantees the existence of the unique maximal local solution $x(t)$ on $\left[-\tau, \sigma_{\infty}\right)$, where $\sigma_{\infty}$ is the explosion time. We need to show $\sigma_{\infty}=\infty$ a.s. If this statement is false, there is a pair of constants $T>0$ and $\epsilon \in(0,1)$ such that

$$
\mathbb{P}\left\{\sigma_{\infty} \leq T\right\}>\epsilon
$$

For each integer $k \geq 1$, define the stopping time

$$
\sigma_{k}=\inf \left\{t \in\left[0, \sigma_{\infty}\right):|x(t)| \geq k\right\}
$$

Since $\sigma_{k} \leq \sigma_{\infty}$, we have

$$
\mathbb{P}\left\{\sigma_{k} \leq T\right\}>\epsilon, \quad \forall k \geq 1
$$

Define

$$
U(y)=\min _{j \in S} V(y, j), \quad \forall y \in \mathbb{R}^{n} .
$$

For any $k \geq 1$ and $0 \leq t \leq T$, by the generalized Itô formula and condition (3.2), we compute

$$
\begin{aligned}
\mathbb{E}\left[V\left(x\left(t \wedge \sigma_{k}\right), r\left(t \wedge \sigma_{k}\right)\right)\right] \leq & \mathbb{E} V\left(\xi(0), r_{0}\right)+h \mathbb{E} \int_{0}^{t \wedge \sigma_{k}}(1+V(x(s), r(s))+U(x(s-\tau))) d s \\
& \left.-\mathbb{E} \int_{0}^{t \wedge \sigma_{k}}(H(x(s))-H(x(s-\tau)))\right) d s \\
\leq & \beta+2 h \int_{0}^{t} \mathbb{E} V\left(x\left(s \wedge \sigma_{k}\right), r\left(s \wedge \sigma_{k}\right)\right) d s \\
\leq & \beta+2 h \int_{0}^{t}\left(\sup _{0 \leq u \leq s} \mathbb{E} V\left(x\left(u \wedge \sigma_{k}\right), r\left(u \wedge \sigma_{k}\right)\right)\right) d s
\end{aligned}
$$

where

$$
\beta=\mathbb{E} V\left(\xi(0), r_{0}\right)+h T+h \tau \sup _{-\tau \leq t \leq 0} \mathbb{E} U(\xi(t))+\tau \sup _{-\tau \leq t \leq 0} \mathbb{E} H(\xi(t))
$$


Since the right-hand-term is increasing in $t$, we must have

$$
\sup _{0 \leq u \leq t} \mathbb{E}\left[V\left(x\left(u \wedge \sigma_{k}\right), r\left(t \wedge \sigma_{k}\right)\right)\right] \leq \beta+2 h \int_{0}^{t}\left(\sup _{0 \leq u \leq s} \mathbb{E} V\left(x\left(u \wedge \sigma_{k}\right), r\left(u \wedge \sigma_{k}\right)\right)\right) d s .
$$

The Gronwall inequality implies

$$
\sup _{0 \leq u \leq T} \mathbb{E}\left[V\left(x\left(u \wedge \sigma_{k}\right), r\left(t \wedge \sigma_{k}\right)\right)\right] \leq \beta e^{2 h T} .
$$

This implies

$$
\mathbb{E}\left[I_{\left\{\sigma_{k} \leq T\right\}} V\left(x\left(\sigma_{k}\right), r\left(\sigma_{k}\right)\right)\right] \leq \beta e^{2 h T} .
$$

On the other hand, if we define

$$
v_{k}=\inf \{V(x, i):|x| \geq k, i \in \mathbb{S}\}
$$

By (3.1), $v_{k} \rightarrow \infty$ as $k \rightarrow \infty$. It now follows from (3.5) and (3.9) that

$$
\beta e^{2 h T} \geq v_{k} \mathbb{P}\left\{\sigma_{k} \leq T\right\} \geq \epsilon v_{k}
$$

Letting $k \rightarrow \infty$ yields a contradiction so we must have $\sigma_{\infty}=\infty$ a.s.

In many practical systems, especially, population systems, we require the existence of the positive global solutions. For this purpose, we define the positive cone $\mathbb{R}_{+}^{n}=\{x \in$ $\left.\mathbb{R}^{n}: x_{i}>0,1 \leq i \leq n\right\}$. Accordingly, we have the following theorem.

Theorem 3.2 Let Assumption 1 hold. Assume that there exists a function $V \in C^{2}\left(\mathbb{R}_{+}^{n} \times\right.$ $\left.\mathbb{S} ; \mathbb{R}_{+}\right)$, a constant $h>0$ and $H \in C\left(\mathbb{R}_{+}^{n} ; \mathbb{R}_{+}\right)$such that

$$
\begin{gathered}
\lim _{x_{j} \rightarrow 0^{+}} V(x, i)=\infty, \quad \lim _{x_{j} \rightarrow \infty} V(x, i)=\infty, \quad \forall i \in \mathbb{S}, \quad j=1, \cdots, n . \\
\mathcal{L} V(x, y, i) \leq h\left(1+V(x, i)+\min _{j \in S} V(y, j)\right)-H(x)+H(y) \quad \forall(x, y, i) \in \mathbb{R}_{+}^{n} \times \mathbb{R}_{+}^{n} \times \mathbb{S} .
\end{gathered}
$$

Assume furthermore that the initial data $\xi \in C_{\mathcal{F}_{0}}\left([-\tau, 0] ; \mathbb{R}_{+}^{n}\right)$ obeys that

$$
\mathbb{E} V\left(\xi(0), r_{0}\right)<\infty, \quad \sup _{-\tau \leq t \leq 0} \mathbb{E}\left[\min _{j \in S} V(\xi(t), j)\right]<\infty, \quad \sup _{-\tau \leq t \leq 0} \mathbb{E} H(\xi(t))<\infty .
$$

Then there exists a unique global solution $x(t)$ to the SDDEwMS (2.1) on $[-\tau, \infty)$ and the solution has the property that $x(t) \in \mathbb{R}_{+}^{n}$ a.s. for all $t \in[-\tau, \infty)$.

Proof. Replacing the definition of stopping time $\sigma_{k}$ in the proof of Theorem 3.1 by

$$
\sigma_{k}=\inf \left\{t \in\left[0, \sigma_{\infty}\right): x_{j}(t) \in(1 / k, k) \text { for some } j=1, \cdots, n\right\}
$$

we can show the theorem in the same way as Theorem 3.1 was proved. 


\section{Approximate Solutions}

Theorem 3.1 gives a very general result on the existence of the unique global solution under the local local Lipschitz condition plus a Lyapunov-type condition. Let us now begin to discuss the EM approximate solutions. The following theorem describes the convergence in probability of the EM solutions to the exact solution under some additional conditions, namely the Lipschtiz condition on the initial data and the local Lipschitz condition on the derivatives of the Lyapunov function.

Theorem 4.1 Let all the assumptions of Theorem 3.1 and Assumption 2 hold. Also assume that for each $R>0$ there exists a positive constant $K_{R}$ such that for all $i \in \mathbb{S}$ and those $x_{1}, x_{2} \in \mathbb{R}^{n}$ with $\left|x_{1}\right| \vee\left|x_{2}\right| \leq R$,

$$
\left|V\left(x_{1}, i\right)-V\left(x_{2}, i\right)\right| \vee\left|V_{x}\left(x_{1}, i\right)-V_{x}\left(x_{2}, i\right)\right| \vee\left|V_{x x}\left(x_{1}, i\right)-V_{x x}\left(x_{2}, i\right)\right| \leq K_{R}\left|x_{1}-x_{2}\right| .
$$

Then for the given initial data $\xi \in L_{\mathcal{F}_{0}}^{2}\left([-\tau, 0] ; \mathbb{R}^{n}\right)$ satisfying (3.3), the exact solution $x(t)$ and the EM approximate solution $X(t)$ to the SDDEwMS (2.1) have the property that

$$
\lim _{\Delta \rightarrow 0}\left(\sup _{0 \leq t \leq T}|X(t)-x(t)|^{2}\right)=0 \quad \text { in probability, for any } T>0 \text {. }
$$

Proof. The proof is rather technical and we divide it into three steps.

Step 1. Fix any $T>0$. For a sufficiently large $R>0$, define the stopping time

$$
\theta=T \wedge \inf \{t \in[0, T]:|x(t)| \geq R\}
$$

Theorem 3.1 tells us that there exists a unique global exact solution $x(t)$ to equation $(2.1)$ on $[-\tau, \infty)$. So

$$
\lim _{R \rightarrow \infty} \mathbb{P}(\theta<T)=0 .
$$

Step 2. Let

$$
v_{R}=\inf \{V(x, i):|x| \geq R, i \in \mathbb{S}\} .
$$

For the sufficiently large $R$ define the stopping time

$$
\rho=T \wedge \inf \{t \in[0, T]:|X(t)| \geq R\} .
$$


Using (2.3) and applying the generalized Itô formula to $V(X(t), r(t))$ yields

$$
\begin{aligned}
& \mathbb{E}[V(X(\rho \wedge t), r(\rho \wedge t))]=\mathbb{E} V\left(\xi(0), r_{0}\right) \\
& +\mathbb{E} \int_{0}^{\rho \wedge t}\left[V_{x}(X(s), r(s)) f(\bar{X}(s), \bar{X}(s-\tau), \bar{r}(s))\right. \\
& +\frac{1}{2} \operatorname{trace}\left[g^{T}(\bar{X}(s), \bar{X}(s-\tau), \bar{r}(s)) V_{x x}(X(s), r(s)) g(\bar{X}(s), \bar{X}(s-\tau), \bar{r}(s))\right] \\
& \left.+\sum_{j=1}^{N} \gamma_{r(s) j} V(X(s), j)\right] d s
\end{aligned}
$$

In the same way as (3.7) was proved, we can show that

$$
\mathbb{E} V\left(\xi(0), r_{0}\right)+\mathbb{E} \int_{0}^{\rho \wedge t} \mathcal{L} V(\bar{X}(s), \bar{r}(s)) d s \leq \beta+2 h \mathbb{E} \int_{0}^{\rho \wedge t} V(\bar{X}(s), \bar{r}(s)) d s,
$$

where $\beta$ is defined by (3.8). Together with (3.2), rearranging the terms on the right-hand side by plus-and-minus technique, we obtain that

$$
\begin{aligned}
\mathbb{E}[ & V(X(\rho \wedge t), r(\rho \wedge t))] \\
\leq \beta & +2 h \mathbb{E} \int_{0}^{\rho \wedge t} V(X(s), r(s)) d s \\
& +2 h \mathbb{E} \int_{0}^{\rho \wedge t}[V(\bar{X}(s), \bar{r}(s))-V(X(s), \bar{r}(s))] d s \\
& +2 h \mathbb{E} \int_{0}^{\rho \wedge t}[V(X(s), \bar{r}(s))-V(X(s), r(s))] d s \\
& +\mathbb{E} \int_{0}^{\rho \wedge t}\left|V_{x}(X(s), r(s))-V_{x}(\bar{X}(s), r(s))\right||f(\bar{X}(s), \bar{X}(s-\tau), \bar{r}(s))| d s \\
& +\mathbb{E} \int_{0}^{\rho \wedge t}\left|V_{x}(\bar{X}(s), r(s))-V_{x}(\bar{X}(s), \bar{r}(s))\right||f(\bar{X}(s), \bar{X}(s-\tau), \bar{r}(s))| d s \\
& +\frac{1}{2} \mathbb{E} \int_{0}^{\rho \wedge t}\left|V_{x x}(X(s), r(s))-V_{x x}(\bar{X}(s), r(s))\right||g(\bar{X}(s), \bar{X}(s-\tau), \bar{r}(s))|^{2} d s \\
& +\frac{1}{2} \mathbb{E} \int_{0}^{\rho \wedge t}\left|V_{x x}(\bar{X}(s), r(s))-V_{x x}(\bar{X}(s), \bar{r}(s))\right||g(\bar{X}(s), \bar{X}(s-\tau), \bar{r}(s))|^{2} d s \\
& +\mathbb{E} \int_{0}^{\rho \wedge t} \sum_{j=1}^{N}\left|\gamma_{r(s) j}\right||V(X(s), j)-V(\bar{X}(s), j)| d s \\
& +\mathbb{E} \int_{0}^{\rho \wedge t} \sum_{j=1}^{N}\left|\gamma_{r(s) j}-\gamma_{\bar{r}(s) j}\right||V(\bar{X}(s), j)| d s .
\end{aligned}
$$


By condition (4.1) we have

$$
\begin{aligned}
& \mathbb{E} \int_{0}^{\rho \wedge t}[V(\bar{X}(s), \bar{r}(s))-V(X(s), \bar{r}(s))] d s \\
\leq & \mathbb{E} \int_{0}^{\rho \wedge t} K_{R}|\bar{X}(s)-X(s)| d s \\
\leq & K_{R} \int_{0}^{T} \mathbb{E}|\bar{X}(\rho \wedge s)-X(\rho \wedge s)| d s \\
\leq & K_{R} \int_{0}^{T}\left(\mathbb{E}|\bar{X}(\rho \wedge s)-X(\rho \wedge s)|^{2}\right)^{\frac{1}{2}} d s .
\end{aligned}
$$

Let $j=[\rho \wedge T / \Delta]$, the integer part of $\rho \wedge T / \Delta$. Then

$$
\begin{aligned}
& \mathbb{E} \int_{0}^{\rho \wedge t}[V(X(s), \bar{r}(s))-V(X(s), r(s))] d s \\
& =\sum_{k=0}^{j} \mathbb{E} \int_{t_{k}}^{t_{k+1}}\left[V\left(X(s), r\left(t_{k}\right)\right)-V(X(s), r(s))\right] d s
\end{aligned}
$$

with $t_{j+1}$ being now set to be $T$. We derive that

$$
\begin{aligned}
& \mathbb{E} \int_{t_{k}}^{t_{k+1}}\left[V\left(X(s), r\left(t_{k}\right)\right)-V(X(s), r(s))\right] d s \\
& \leq \mathbb{E} \int_{t_{k}}^{t_{k+1}}\left[V\left(X(s), r\left(t_{k}\right)\right)-V(X(s), r(s))\right] I_{\left\{r(s) \neq r\left(t_{k}\right)\right\}} d s \\
& \leq 2 V_{R} \mathbb{E} \int_{t_{k}}^{t_{k+1}} I_{\left\{r(s) \neq r\left(t_{k}\right)\right\}} d s \\
& =2 V_{R} \int_{t_{k}}^{t_{k+1}} \mathbb{E}\left(I_{\left\{r(s) \neq r\left(t_{k}\right)\right\}} \mid r\left(t_{k}\right)\right) d s,
\end{aligned}
$$

where $V_{R}=\max \{V(x, i):|x| \leq R, i \in \mathbb{S}\}$ and in the last step we use the fact that $I_{\left\{r(s) \neq r\left(t_{k}\right)\right\}}$ is conditionally independent with respect to the $\sigma$-algebra generated by $r\left(t_{k}\right)$. But, by the Markov property,

$$
\begin{aligned}
\mathbb{E}\left(I_{\left\{r(s) \neq r\left(t_{k}\right)\right\}} \mid r\left(t_{k}\right)\right) & =\sum_{i \in \mathbb{S}} I_{\left\{r\left(t_{k}\right)=i\right\}} \mathbb{P}\left(r(s) \neq i \mid r\left(t_{k}\right)=i\right) \\
& =\sum_{i \in \mathbb{S}} I_{\left\{r\left(t_{k}\right)=i\right\}} \sum_{j \neq i}\left(\gamma_{i j}\left(s-t_{k}\right)+o\left(s-t_{k}\right)\right) \\
& \leq\left(\max _{1 \leq i \leq N}\left(-\gamma_{i i}\right) \Delta+o(\Delta)\right) \sum_{i \in \mathbb{S}} I_{\left\{r\left(t_{k}\right)=i\right\}} \\
& \leq \bar{\gamma}(\Delta+o(\Delta)),
\end{aligned}
$$

where $\bar{\gamma}=\max _{1 \leq i \leq N}\left(-\gamma_{i i}\right)$. So, inequalities (4.6)-(4.8) imply

$$
\mathbb{E} \int_{0}^{\rho \wedge t}[V(X(s), \bar{r}(s))-V(X(s), r(s))] d s \leq 2 V_{R} T \bar{\gamma}(\Delta+o(\Delta)) .
$$


We can similarly estimate the other terms on the right-hand side of (4.5) to get that

$$
\begin{aligned}
\mathbb{E}[V(X(\rho \wedge t), r(\rho \wedge t))] & \leq \beta+2 h \mathbb{E} \int_{0}^{\rho \wedge t} V(X(s), r(s)) d s \\
& +C_{1}(R) \int_{0}^{T}\left(\mathbb{E}|\bar{X}(\rho \wedge s)-X(\rho \wedge s)|^{2}\right)^{\frac{1}{2}} d s \\
& +C_{1}(R)(\Delta+o(\Delta)),
\end{aligned}
$$

where $C_{1}(R)$ and the following $C_{2}(R), C_{3}(R), \cdots$ are all constants dependent of $R$ but independent of $\Delta$. But, for $s \in[0, T]$, let $k_{s}=[\rho \wedge s / \Delta]$, the integer part of $\rho \wedge s / \Delta$. It follows from (2.3) and Assumption 1 easily that

$$
\mathbb{E}|\bar{X}(\rho \wedge s)-X(\rho \wedge s)|^{2} \leq C_{2}(R) \Delta \quad \forall s \in[0, T]
$$

Substituting this into (4.9) yields that

$$
\mathbb{E}[V(X(\rho \wedge t), r(\rho \wedge t))] \leq \beta+C_{3}(R)\left(\Delta^{\frac{1}{2}}+o\left(\Delta^{\frac{1}{2}}\right)\right)+2 h \int_{0}^{t} \mathbb{E} V(X(\rho \wedge s), r(\rho \wedge s)) d s .
$$

By the Gronwall inequality,

$$
\mathbb{E}[V(X(\rho \wedge T), r(\rho \wedge T))] \leq e^{2 h T}\left[\beta+C_{3}(R)\left(\Delta^{\frac{1}{2}}+o\left(\Delta^{\frac{1}{2}}\right)\right)\right]
$$

In the same way as (3.11) was obtained, we can then show that

$$
P(\rho<T) \leq \frac{e^{2 h T}}{v_{R}}\left[\beta+C_{3}(R)\left(\Delta^{\frac{1}{2}}+o\left(\Delta^{\frac{1}{2}}\right)\right)\right] .
$$

Step 3. Let $\tau=\rho \wedge \theta$. In the same way as Theorem 7.29 of [7] was proved we can show that

$$
\mathbb{E}\left[\sup _{0 \leq t \leq \tau \wedge T}|X(t)-x(t)|^{2}\right] \leq C_{4}(R)(\Delta+o(\Delta))
$$

We would like to remark that Assumption 2 is used here. Now, let $\varepsilon, \delta \in(0,1)$ be arbitrarily small. Set

$$
\bar{\Omega}=\left\{\omega: \sup _{0 \leq t \leq T}|X(t)-x(t)|^{2} \geq \delta\right\} .
$$

Using (4.12), we compute

$$
\begin{aligned}
\delta \mathbb{P}(\bar{\Omega} \cap\{\tau \geq T\}) & =\delta \mathbb{E}\left[I_{\{\tau \geq T\}} I_{\bar{\Omega}}\right] \\
& \leq \mathbb{E}\left[I_{\{\tau \geq T\}} \sup _{0 \leq t \leq \tau \wedge T}|X(t)-x(t)|^{2}\right] \\
& \leq \mathbb{E}\left[\sup _{0 \leq t \leq \tau \wedge T}|X(t)-x(t)|^{2}\right] \\
& \leq C_{4}(R)(\Delta+o(\Delta)) .
\end{aligned}
$$


This, together with (4.11), yields that

$$
\begin{aligned}
\mathbb{P}(\bar{\Omega}) & \leq \mathbb{P}(\bar{\Omega} \cap\{\tau \geq T\})+\mathbb{P}(\tau<T) \\
& \leq \mathbb{P}(\bar{\Omega} \cap\{\tau \geq T\})+\mathbb{P}(\theta<T)+\mathbb{P}(\rho<T) \\
& \leq \frac{C_{4}(R)}{\delta}(\Delta+o(\Delta))+\mathbb{P}(\theta<T)+\frac{e^{2 h T}}{v_{R}}\left[\beta+C_{3}(R)\left(\Delta^{\frac{1}{2}}+o\left(\Delta^{\frac{1}{2}}\right)\right)\right] .
\end{aligned}
$$

Recalling (4.3) and that $v_{R} \rightarrow \infty$ as $R \rightarrow \infty$, we can choose $R$ sufficiently large for

$$
\mathbb{P}(\theta<T)<\frac{\varepsilon}{3}, \quad \frac{\beta e^{2 h T}}{v_{R}}<\frac{\varepsilon}{3},
$$

and then choose $\Delta$ sufficiently small for

$$
\frac{C_{4}(R)}{\delta}(\Delta+o(\Delta))+\frac{e^{2 h T}}{v_{R}} C_{3}(R)\left(\Delta^{\frac{1}{2}}+o\left(\Delta^{\frac{1}{2}}\right)\right)<\frac{\varepsilon}{3}
$$

to obtain

$$
\mathbb{P}(\bar{\Omega})=\mathbb{P}\left(\sup _{0 \leq t \leq T}|X(t)-x(t)|^{2} \geq \delta\right)<\varepsilon .
$$

This proves the assertion (4.2).

Similarly, we can show the EM approximate solutions will converge to the exact positive solution under the conditions of Theorem 3.2 along with Assumption 2 and condition (4.1) which is of course restricted in the positive cone.

\section{Application to the Stochastic Hybrid Delay Popu- lation System}

Let us now return to the stochastic hybrid delay population system (1.2). Clearly, the coefficients satisfy Assumption 1. Yuan et al. [13] found a suitable Lyapunov function for this system

$$
V(x, i)=\sum_{j=1}^{n} c_{m}(i)\left(x_{j}-1-\ln \left(x_{j}\right)\right), \quad(x, i) \in \mathbb{R}_{+}^{n} \times \mathbb{S},
$$

for $n N$ positive constants $c_{1}(i), \cdots, c_{n}(i)$ for $i \in \mathbb{S}$. Obviously, this function satisfies conditions (3.12) and (4.1) (restricted in the positive cone). Yuan et al. [13] imposed the following assumption:

Assumption 3 Assume that there exist $n N+1$ positive constants $c_{1}(i), \cdots, c_{n}(i)$ for $i \in \mathbb{S}$ and $\theta$ such that

$$
\lambda_{\max }^{+}\left(\frac{1}{2}\left[\bar{C}(i) A(i)+A^{T}(i) \bar{C}(i)\right]+\frac{1}{4 \theta} C(i) B(i) B^{T}(i) C(i)+\theta I\right) \leq 0,
$$


where $\bar{C}(i)=\operatorname{diag}\left(c_{1}(i), \cdots, c_{n}(i)\right)$ and $I$ is the $n \times n$ identity matrix.

Under Assumption 3 the authors showed that there is a constant $h>0$ such that

$$
\mathcal{L} V(x, y, i) \leq h\left(1+V(x, i)+\min _{j \in \mathbb{S}} V(y, j)\right)-\theta|x|^{2}+\theta|y|^{2}, \quad \forall(x, y, i) \in \mathbb{R}_{+}^{n} \times \mathbb{R}_{+}^{n} \times \mathbb{S} .
$$

Therefore, Theorem 3.2 shows that for any initial data $\xi \in C_{\mathcal{F}_{0}}^{b}\left([-\tau, 0] ; \mathbb{R}^{n}\right)$ and $r(0)=$ $r_{0} \in L_{\mathcal{F}_{0}}(\Omega ; \mathbb{S})$, there is a unique global solution $x(t) \in \mathbb{R}_{+}^{n}$ to equation (1.2) on $t \in$ $[-\tau, \infty)$. Moreover, the EM scheme will converge to the true solution $x(t)$ in the sense of Theorem 4.1. So we could make extensive use of simulations of (1.2) to confirm analytic results and to explore model behaviour.

\section{References}

[1] N. H. Du, R. Kon, K. Sato and Y. Takeuchi, Dynamical Behavior of Lotka-Volterra Competition Systems: Non-autonomous Bistable Case and the Effect of Telegraph Noise, J. Comput. Appl. math., 170(2004), 399-422.

[2] D. J. Higham, X. Mao and A.M. Stuart, Strong convergence of numerical methods for nonlinear stochastic differential equations, SIAM J. Numer. Anal., 40 (2002) 10411063.

[3] P. E. Kloeden and E. Platen, Numerical Solution of Stochastic Differential Equations, Spinger-Verlag, Berlin, 1999.

[4] Q. Luo and X. Mao, Stochastic population dynamics under regime switching, J. Math. Anal. Appl., 334(2007), 69-84.

[5] X. Mao, Stochastic Differential Equations and Applications, 2nd Edition, Horwood, Chichester, 2007.

[6] X. Mao, Y. Shen and C. Yuan, Almost surely asymptotic stability of neutral stochastic differential delay equations with Markovian switching, Stochastic Processes and their Applications, 118(2008), 1385-1406.

[7] X. Mao and C. Yuan, Stochastic differential equations with Markovian switching, Imperial College Press, 2006. 
[8] M. Mariton, Jump Linear Systems in Automatic Control, Marcel Dekker, NewYork, 1990.

[9] G. Marion, X. Mao and E. Renshaw, Convergence of the Euler scheme for a class of stochastic differential equation, Inern. Math. Journal, 1(2002), 9-22.

[10] A. V. Skorohod, Asymptotic Methods in the Theory of Stochastic Differential Equations, American Mathematical Society, Providence, RI, 1989.

[11] M. Slatkin, The Dynamics of a Population in a Markovian Environment, Ecology, 59(1978), 249-256.

[12] Y. Takeuchi, N. H. Du, N. T. Hieu and K. Sato, Evolution of predator-prey systems described by a Lotka-Volterra equation under random environment, J. Math. Anal. Appl., 323 (2006), 938-957.

[13] C. Yuan, X. Mao and J. Lygeros, Stochastic hybrid delay population dynamics: well posed models and extinction, in press. 